\title{
Effects of postural changes of the upper limb on reflex transmission in the lower limb
}

\section{Cervicolumbar reflex interactions in man}

\author{
P. J . D E L W A I D E, C. F I G I E L, A N D C. R I C H E L L E \\ From the Section of Neurology and Clinical Neurophysiology, Department of Internal Medicine, \\ University of Liege, Liege, Belgium
}

SUMMARY The influence of passive changes in upper limb position on the excitability of three myotatic arc reflexes (soleus, quadriceps, and biceps femoris) of the lower limb has been explored on 42 volunteers. The results indicate that the excitability of the three myotatic arcs can be influenced at a distance by postural modifications of the upper limb. When the ipsilateral upper limb is forwards or the contralateral backwards, a facilitation of both soleus and quadriceps tendon reflexes is observed while the biceps femoris reflexes are reduced. This pattern of facilitation and inhibition is reversed when the ipsilateral upper limb is backwards or the contralateral forwards. The facilitations as well as inhibitions of proximal myotatic arc reflexes are quantitatively more marked than that of the soleus reflex. Facilitation and inhibition are not linearly related to the angle of the arm with the trunk. Effects begin at a considerable angle, become maximal at $45^{\circ}$, and progressively disappear for greater values. It is suggested that the distinct pattern of facilitation and inhibition which is exerted in reciprocal fashion on extensor and flexor motor nuclei might depend on the long propriospinal neurones connecting cervical and lumbar enlargements.

Some aspects of muscular coordination between the various myotatic arcs of a limb in man have been studied (Delwaide et al., 1976). In this case, segmental spinal mechanisms are supposed to play an important role. Long, intersegmental coordination should however be emphasised.

In the cat, cervicolumbar reflex interactions are well known (Lloyd, 1942; Lloyd and McIntyre, 1948; Djalali, 1970). These interactions have a great interest in relation to gait mechanisms: the movements of the upper and lower limbs are coordinated in walking, and it has been shown that this coordination is brought about, at least in part, by reflex systems within the spinal cord. In man, because of the bipedal position, it is a matter of speculation as to what extent the activities of the upper and lower limbs are coordinated. If a specific influence of arm swing in natural walking

Address for correspondence and reprint requests: Dr P. J. Delwaide, Section of Neurology and Clinical Neurophysiology, Department of Internal Medicine, Hôpital de Bavière, Bd de la Constitution 66, 4020, Liege, Belgium.

Accepted 24 December 1976 may be supposed to be only a vestigial function, some physiological links between upper and lower limbs may be looked for in other circumstances. For example, when the arm is horizontal instead of vertical, some changes in tone and posture may be suspected, especially in the leg, to adjust the equilibrium of the body.

In this study, a possible influence of passive changes in upper limb position on the excitability of motoneurones supplying lower limb musculature has been explored. We have considered three myotatic arcs activated by their tendon reflexes. Soleus, quadriceps, and biceps femoris myotatic arcs were selected. We have tried to establish whether there is a different pattern of effects in extensor and flexor, proximal or distal muscles in relation to changes in position of ipsilateral and contralateral upper limbs respectively. Preliminary results dealing only with the soleus tendon reflex have been published (Delwaide et al., 1973).

\section{Sample and methods}

Forty-two volunteers, mostly students, aged from 
16 to 27 years, have been investigated in a total of 82 experiments. The subjects were placed in a seated position with the trunk inclined backwards at $30^{\circ}$. Both lower limbs were kept parallel by fixation of the knee and foot, such that the knee was flexed at $135^{\circ}$ and the ankle plantar-flexed to $110^{\circ}$. The head was immobilised.

The soleus, quadriceps, and biceps femoris myotatic reflexes were evoked by percussion of their respective tendons by firmly fixed electromagnetic hammers. The intensity as well as the angle of each percussion was similar. In every case, the maximal reflex response in resting conditions was sought.

Disa 13K60 cutaneous electrodes were fixed over the three lower limb muscles under investigation. Electromyographic activity was amplified by Tektronix 3A9 plug-in amplifiers, displayed on a Tektronix 565 oscilloscope, and measured on a digital oscilloscope (Tektronix Type 230 Digital Unit), while the values were printed out by a Hewlett Packard 5050B. Further cutaneous electrodes were fixed over the following muscles of the shoulder girdle and upper limb: deltoid, biceps, and pectoralis major. In each case it was verified that there was no spontaneous electromyographic activity in the muscles under investigation, in either the upper or the lower limb.

The reference value for each reflex corresponded to the mean of results obtained when both arms lay along the thoracic wall in the midaxillary line. These values were compared with those obtained when one of the two upper limbs with the forearm in extension was at various angles, either towards the front (anteflexion) or towards the back (extension) relative to the starting position. The displacement was passive. At every angle, a comfortable support maintained the position of the upper limb without any electromyographic activity being detected in those of its muscles from which recordings were made. For each position tested, an average of 30 to 50 nonconsecutive measurements of lower limb amplitudes were made, in order to avoid the influence of global variations in reflex activity, and of general factors such as somnolence. In practice, series of five reflexes were evoked alternately in the reference and test positions of the upper limb. The reflexes were evoked at intervals of seven seconds.

\section{Results}

INVESTIGATION OF THE SOLEUS MYOTATIC ARC

Schematically, four different situations can be distinguished according to whether the ipsilateral or the contralateral arm respectively is angled forwards or backwards. Figure 1 shows the results obtained in each of these situations. Each of the 82 points on this figure represents the mean of 30 measurements. The results are expressed as a percentage of the reference values obtained when both arms were in the mid-axillary line. In each of the situations studied, the upper limb with the forearm in extension was placed either $60^{\circ}$ towards the front or $30^{\circ}$ towards the back.

\section{Soleus Tendon Reflex}

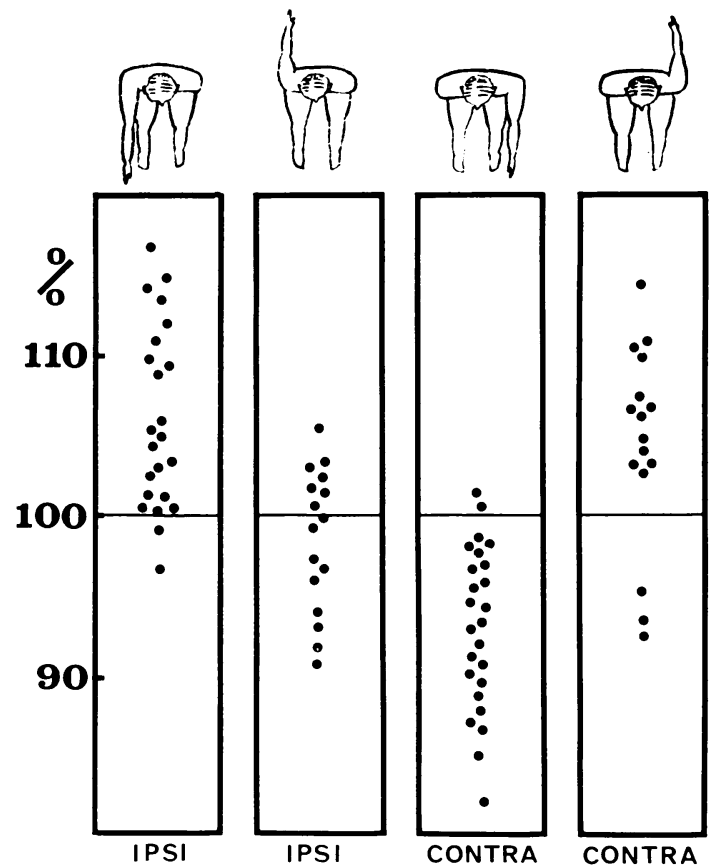

Fig. 1 Amplitude of soleus tendon reflex expressed as percentage of reference values obtained with arms in mid-axillary line (each point is mean of 30 measurements). Reflex evoked from right calf. Values are increased when the ipsilateral arm is anteflexed and the contralateral arm extended; they are reduced when the ipsilateral arm is extended and the contralateral arm anteflexed.

It can be seen that in each of the four situations represented in Fig. 1, most amplitudes of the tendon reflexes differed from the reference values. However, the individual values were scattered and the variations from the reference values were relatively small. Statistical evaluation (comparison of the two means and calculation of the reduced differences) indicates that the differences were 
significant when either arm was in the forward position. When the arm ipsilateral to the leg under test was flexed $60^{\circ}$ forwards, there was an average increase in the amplitude of the tendon reflex, while when it was extended backwards at $30^{\circ}$, a reduction in reflex amplitude was observed. When the contralateral arm was forwards, inhibition rather than facilitation of the soleus reflex was found, while backward extension brought about facilitation.

The mean value of the facilitation when the ipsilateral arm was anteflexed was $6.9 \%$, while when the contralateral arm was in the same position, the inhibition reached a mean value very close to $6.8 \%$. Despite the dispersion of values in the population of test subjects, the same experimental manoeuvre gave rise to fairly similar results when it was repeated on the same individual after an interval of several weeks. For example, from subject GJ, tested four times, the following values for facilitation were obtained when the ipsilateral arm was anteflexed: $+9.2 \%,+9.6 \%$, $+9.8 \%$, and $+11.2 \%$; when the contralateral arm was anteflexed: $-10 \%,-9.4 \%,-9.1 \%$, and $-11 \%$.

The variations in the amplitude of the soleus tendon reflex are a function of the angle between the raised arm and the trunk (Fig. 2). Curve A shows the values measured when the arm ipsilateral to the leg under test was anteflexed successively to $15,30,45,60,75$, and $90^{\circ}$ relative to the trunk. At an angle of $15^{\circ}$, no alteration in the soleus reflex was noted. A slight difference appeared at $30^{\circ}$, while at $45^{\circ}$ it was clear, and indeed maximal. The effect did not increase, but was reduced, when the arm was $60^{\circ}$ forwards, and disappeared at $90^{\circ}$.

When the inhibition, rather than the facilita-

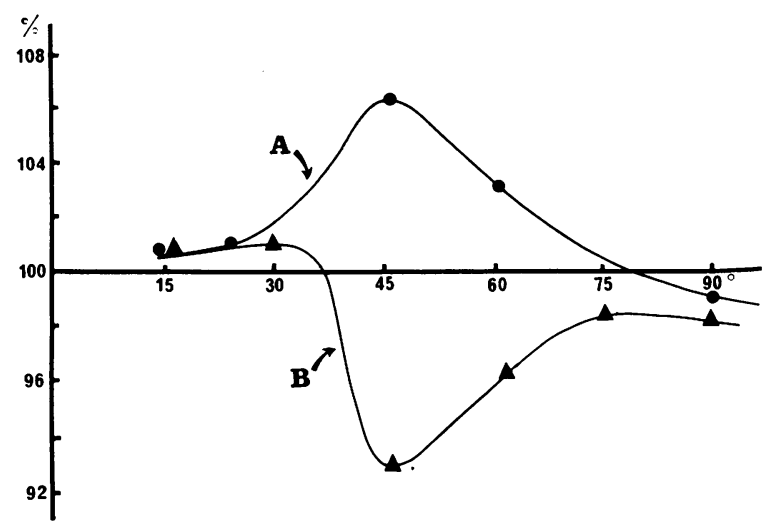

tion, brought about by the contralateral arm was examined, the same effects appeared as a function of the angulation of the arm (Fig. 2B). No effect was seen at $15^{\circ}$ or even at $30^{\circ}$; the effect was maximal at $45^{\circ}$ and became progressively reduced up to $90^{\circ}$. When two facilitatory manoeuvres, such as flexion of the ipsilateral and extension of the contralateral arm, were performed simultaneously, the effect measured was usually greater than when either was carried out alone. Although marked individual variations occurred, the combination sometimes reached a value equal to the sum of the two obtained separately. Conversely, simultaneous performance of inhibitory manoeuvres (anteflexion of contralateral and extension of ipsilateral arm) brought about a greater inhibitory effect. This latter result is particularly interesting since it indicates that the results are not due to a non-specific phenomenon such as the Jendrassik manoeuvre.

INVESTIGATION OF THE QUADRICEPS MYOTATIC ARC The amplitude of the quadriceps tendon reflex is also modified when a comparison is made between reference values and those obtained with a raised arm in various positions.

When the ipsilateral upper limb was forwards or the contralateral backwards, a clear facilitation of the quadriceps tendon reflex was observed. On the other hand, when the contralateral arm was forwards or the ipsilateral backwards, inhibition was observed. Thus the quadriceps reflex was influenced by the various conditioning positions of the upper limb in the same direction as was the soleus reflex.

As is shown in Fig. 3, the intensity of the effect is related to the angulation of the arm. For an angle of $15^{\circ}$ forwards, there was no effect; at $30^{\circ}$,

Fig. 2 Variations in amplitude of the Achilles tendon reflex as a function of the angle between arm and trunk. Amplitude is expressed as percentage of reference values obtained with both arms in the mid-axillary line. Curve $A$ shows values obtained when the ipsilateral arm is at various forward angles. Curve $B$ shows values obtained when the contralateral arm is at various forward angles. 

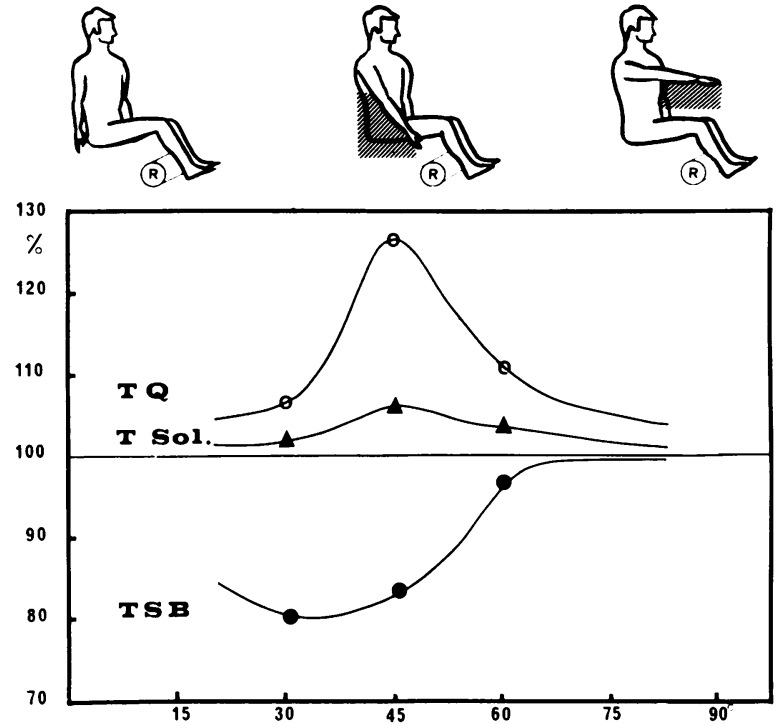

Fig. 3 Changes of amplitude in quadriceps $(T Q)$, soleus (T Sol.), and short head of biceps femoris (TSB) tendon reflexes at different stepped forward angles of the ipsilateral upper limb. Values are expressed as a percentage of reference measurements made with both arms in the mid-axillary line. Facilitation of the quadriceps tendon reflex is more marked than that of the soleus. The short head of biceps reflex is inhibited. mean facilitation was $7 \%$, at $45^{\circ}, 28 \%$, while at $60^{\circ}$ it was reduced to $11 \%$. The facilitation of the quadriceps was quantitatively more marked than that of the soleus reflex; the mean value of $+28 \%$ observed when the ipsilateral arm was anteflexed to $45^{\circ}$ contrasted with the $+6 \%$ facilitation seen in the soleus.

The inhibition measured when the contralateral arm was anteflexed was also marked. As is shown in Fig. 4, clear inhibition already occurred at an angle of $30^{\circ}$, and maintained a relatively stable level between $30^{\circ}$ and $60^{\circ}$.

INVESTIGATION OF THE BICEPS FEMORIS MYOTATIC ARC

Contrary to the results obtained from an examination of the (extensor) soleus and quadriceps reflexes, forward flexion of the ipsilateral arm induced an inhibition of the tendon reflex in the biceps, which is a flexor. Figure 3 illustrates this
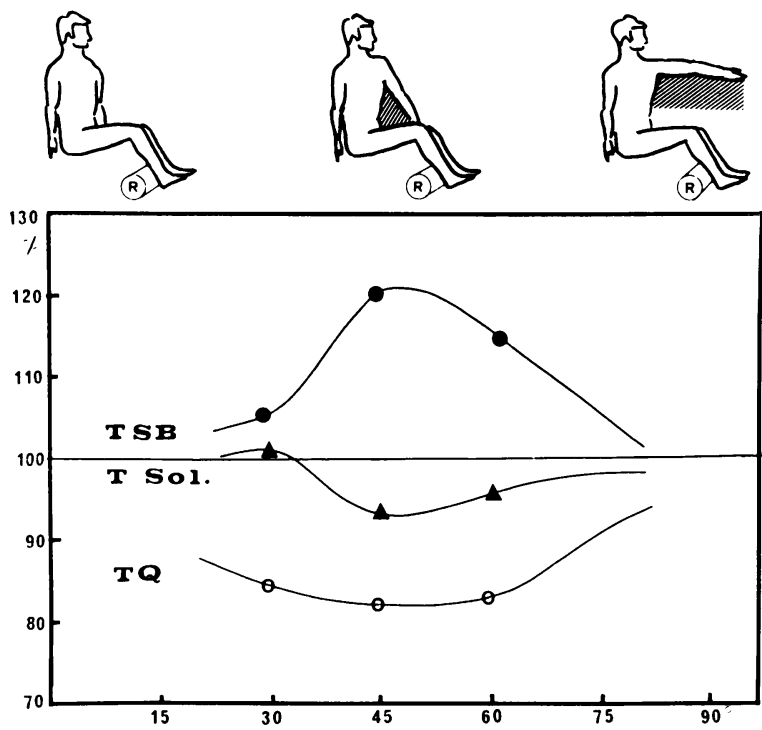

Fig. 4 Changes of amplitude in quadriceps $(T Q)$, soleus (T Sol.), and short head of biceps femoris (TSB) tendon reflexes at different stepped forward angles of the contralateral arm. In this situation, the short head of biceps femoris tendon reflex is facilitated while those of quadriceps and soleus are reduced. 
inhibitory effect, which was already apparent at an angle of $30^{\circ}$, reaching $20 \%$. It was marked at $45^{\circ}$, but subsequently greatly reduced at $60^{\circ}$.

When the contralateral arm was anteflexed, facilitation was seen (Fig. 4). This was influenced by the angle obtained between the arm and the thorax. Already present at $30^{\circ}$, it reached a maximum of $20 \%$ at $45^{\circ}$, and was then progressively reduced until it disappeared at $90^{\circ}$.

When the ipsilateral arm was extended, facilitation occurred, while inhibition took place when the contralateral arm was extended.

\section{Discussion}

The results reported above indicate that the excitability of the three myotatic arcs of the lower limb in man can be influenced at a distance by postural modification of the upper limbs. The latter are responsible for a distinct pattern of facilitation and inhibition which are exerted in reciprocal fashion on extensor (soleus and quadriceps) and flexor (biceps femoris) muscles.

The distant effect is exerted by a specific mechanism quite distinct from that which is brought into play by the Jendrassik manoeuvre. In the first place, electromyographic recordings indicate the absence of muscular activity in the upper limb whose displacement influences lower limb reflexes. Furthermore, even if a Jendrassik effect due to an unnoticed muscular contraction could be invoked to explain reflex facilitation, it could not account for the inhibitory effects demonstrated. Voluntary contraction in a distant muscle increases the excitability of all myotatic reflexes in the lower limb (Delwaide et al., to be published).

Facilitation and inhibition are discrete effects in the soleus reflex arc. One may speculate about their functional value. Are they perhaps more intense in the presence of distant motor activity than in conditions of passive postural change? Further investigations are required on this point. On the other hand, even in repose, the effects are clearly more marked in the thigh muscle than in the calf. The greater intensity of the effect on the proximal limb segment has also been observed in a study of the effect of caloric stimulation of the vestibular apparatus on lower limb myotatic reflexes (Delwaide, 1977), and in an investigation of the Jendrassik manoeuvre (Delwaide $e t$ al., to be published). These findings should perhaps be correlated with anatomofunctional data on descending spinal pathways.

Facilitation and inhibition are not related linearly to the angle of the arm with the trunk, but in some more complex way. When the arm is anteflexed, effects begin at a considerable angle and become maximal at $45^{\circ}$. Beyond this angle they are reduced, disappearing when the arm forms an angle of $90^{\circ}$ with the trunk. The curve could possibly be the result of actions in opposite directions evoked by the discharge of receptors possessing different recruitment thresholds. It can be noted that most evident effects are observed in the range of upper limb displacements occurring during walking (Ballesteros et al., 1965).

To account for the effects of postural changes of the upper limb on reflex transmission in the lower limb, the long propriospinal neurones connecting cervical and lumbar enlargements of the spinal cord deserve special attention. In the cat, they seem to play an important role in forelimb-hindlimb coordination during walking (Miller and Van Der Burg, 1973). Long propriospinal fibres have monosynaptic excitatory connections with many motoneurones and with excitatory and inhibitory interneurones projecting to motoneurones (Jankowska et al., 1974). So, the propriospinal circuitry could possibly be the way by which the distinct pattern of facilitation and inhibition is exerted on lumbar neurones. Moreover, the neurones activated by the propriospinal tract are almost if not exclusively confined to the ventral horn, and especially to its medial aspects. A more prominent effect on proximal myotatic arcs could be interpreted by this fact.

At present, preliminary findings indicate synaptic activation from the brain of the long propriospinal neurones. In awake normal subjects, it is likely that higher centres contribute with spinal structures to influence the reflex transmission in the lower limb. In the cat, a proprioceptive receiving area of the cerebral cortex has been shown to enhance the cervicolumbar interactions (Abrahams and Falchetto, 1969; Abrahams, 1970).

However, before taking as proven that cervicolumbar interactions in man depend almost entirely on a propriospinal system, the physiology of this structure and of its control has to be better understood.

\section{References}

Abrahams, V. C. (1970). Cervico-lumbar reflex interactions involving a proprioceptive receiving area of the cerebral cortex. Journal of Physiology, 209, 45-46.

Abrahams, V. C. and Falchetto, S. (1969). Hind leg ataxia of cervical origin and cervico-lumbar spinal interactions with a supratentorial pathway. Journal 
of Physiology, 203, 435-447.

Ballesteros, M. L., Buchthal, F., and Rosenfalck, P. (1965). The pattern of muscular activity during the arm swing of natural walking. Acta Physiologica Scandinavica, 63, 296-310.

Delwaide, P. J. (1977). Excitability of lower limb myotatic arcs under influence of caloric labyrinthine stimulation: Analysis of the postural effects in man. Journal of Neurology, Neurosurgery, and Psychiatry. In press.

Delwaide, P. J., Figiel, C., and Richelle, C. (1973). Influence de la position du membre supérieur sur l'excitabilité de l'arc soléaire. Electromyography and Clinical Neurophysiology, 13, 515-523.

Delwaide, P. J., Toulouse, P., and Juprelle, M. (1976). Quantitative aspects of the Jendrassik's manoeuvre in man. In press.

Djalali, E. (1970). Relations neurophysiologiques brachio-lombaires chez le chat spinal. Contribution à la connaissance de la physiologie de la moelle épinière. Thése Faculté des Sciences de l'Université d'Aix Marseille-CNRS, $150 \mathrm{p}$.

Jankowska, E., Lundberg, A., Roberts, W. J., and Stuart, D. (1974). A long propriospinal system with direct effect on motoneurones and on interneurones in the cat lumbosacral cord. Brain Research, 21, 169-194.

Lloyd, D. P. C. (1942). Mediation of descending long spinal reflex activity. Journal of Neurophysiology, $5,435-458$.

Lloyd, D. P. C., and McIntyre, A. K. (1948). Analysis of forelimb-hindlimb reflex activity in acutely decapitate cats. Journal of Neurophysiology, 11, 455470.

Miller, S., and Van Der Burg, J. (1973). The function of long propriospinal pathways in the coordination of quadrupedal stepping in the cat. In Control of Posture and Locomotion. Edited by R. B. Stein, K. G. Pearson, R. S. Smith, and J. B. Redford. Pp. 561-578. Plenum Press: New York. 